\title{
Применение локального
}

\section{длинноволнового инфракрасного излучения в комплексной терапии ограниченной склеродермии}

\author{
Секирин А.Б. • Смирнова С.Н. • Сухова Т.Е.' • Майбродская А.Е.
}

Актуальность. Ограниченная склеродермия часто приводит к необратимым косметическим изменениям, а в далеко зашедших случаях к нарушению функции органа и инвалидности. Медикаментозная терапия недостаточно эффективна, она может вызывать побочные явления и осложнения. В связи с этим большое внимание уделяется немедикаментозным методам лечения данного заболевания, способным оказывать положительное клинико-патогенетическое влияние и потенцировать эффекты медикаментозной терапии. Цель - изучение влияния локального длинноволнового инфракрасного излучения на течение ограниченной склеродермии и оценка терапевтической эффективности данного метода в комплексе с традиционно проводимой медикаментозной терапией. Материал и методы. Под наблюдением находились 55 пациентов с ограниченной склеродермией. Пациенты контрольной группы $(\mathrm{n}=20)$ в течение 14-20 суток получали стандартную медикаментозную терапию: антибактериальные препараты, протеолитические ферменты, блокаторы кальциевых каналов, антиоксиданты, ангиопротекторы и корректоры микроциркуляции для местного применения, а также аппликации кортикостероидных гормонов. Пациентам основной группы $(n=35)$ на фоне стандартной медикаментозной терапии проводилось локальное длинноволновое инфракрасное облучение патологических очагов (длина волны 4-16 мкм, 1 раз в сутки 10 дней). Оценивали динамику интенсивности отека и гиперемии по периферии очага поражения, распространенность и выраженность патологического процесса с помощью модифицированной методики балльной системы MRODNAN Skin Score, а также состояние микроциркуляторного русла на аппарате «Спектротест». Результаты. У пациентов, получавших в дополнение к стандартной медикаментозной терапии ограниченной склеродермии локальное длинноволновое инфракрасное излучение, по сравнению с контрольной группой было отмечено более выраженное снижение индекса mRODNAN $(6,3 \pm 0,5$ и 2,2 $\pm 0,1$ соответственно, $\mathrm{p}<0,05)$, значительное снижение показателей сатурации кислорода в патологических очагах $(0,9 \pm 0,001$ и $0,646 \pm 0,04$ у.е., $\mathrm{p}<0,05)$ и повышение объема кровотока В микроциркуляторном русле $(0,091 \pm 0,002$ и 0,23 $\pm 0,045$ у.е., $p<0,05)$, а также более быстрое наступление клинического эффекта - на $14 \pm 2,3$ дня $(p<0,05)$. В контрольной группе клинический эффект наступал лишь на $20 \pm 4,2$ дня $(p<0,05)$, в очагах поражения была отмечена тенденция к снижению сатурации кислорода

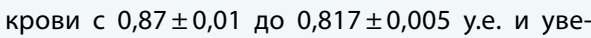
личению объема кровотока в микроциркуляторном русле с $0,086 \pm 0,004$ до $0,1 \pm 0,003$ у.е. Стандартная медикаментозная терапия не обеспечила полного разрешения элементов ограниченной склеродермии, что подтверждалось результатами оценки индекса mRODNAN (до лечения его среднее значение в этой группе составляло $6,4 \pm 0,5$, после лечения $-5,3 \pm 0,2$, $p \geq 0,05)$. Заключение. Доказана клиническая эффективность применения локального длинноволнового инфракрасного излучения в комплексной терапии ограниченной склеродермии. Положительный эффект выразился В нормализации показателей микроциркуляции, увеличении показателей кровотока, снижении показателей сатурации кислорода в очагах поражения (развитие активной репарации), а также повышении клинической эффективности и ее более быстром наступлении.

Ключевые слова: ограниченная склеродермия, инфракрасное излучение, физиотерапия, эффективность

doi: 10.18786/2072-0505-2016-44-1-28-32 


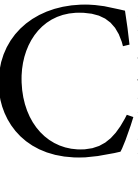

клеродермия - хроническое прогрессирующее заболевание из группы коллагенозов, характеризующееся ограниченным или диффузным поражением кожи в виде ее уплотнения с последующим развитием атрофии. Выделяют системную (с поражением внутренних органов) и ограниченную кожей склеродермию. Этиология и патогенез склеродермии не установлены. В последнее время ее рассматривают как аутоиммунное заболевание соединительной ткани. Однако теории инфекционного, нейроэндокринного и сосудистого генеза этого процесса также не утратили своего значения [1]. Заболеваемость составляет в среднем 8-12 случаев на 1 млн населения. Преимущественно болеют женщины трудоспособного возраста (30-40 лет) в соотношении 3-4 на 1 заболевшего мужчину [2]. Прогрессирование ограниченной формы склеродермии в системную встречается крайне редко - 0,9-5,7\% пациентов [3]. При стандартной медикаментозной терапии прогноз для жизни благоприятный, для полного излечения - сомнительный. Применение системных кортикостероидов и иммуносупрессантов, обусловленное аутоиммунной теорией возникновения заболевания, сопровождается большим количеством нежелательных последствий [4]. Без эффективного лечения ограниченная склеродермия нередко приводит к необратимым косметическим изменениям (рис. 1), а в далеко зашедших случаях - к нарушению функции органа и даже к инвалидности.

Благоприятное влияние на течение данного заболевания оказывают широко известные и хорошо изученные физиотерапевтические методы, например, такие как электрофорез ферментных препаратов, ультразвуковая терапия и ультрафонофорез кортикостероидов, микроволновая терапия, грязевые, озокеритовые и парафиновые аппликации, вибро- и гидромассаж [5]. К более современным методам относятся лазерная терапия, ПУВА-терапия, длинноволновое ультрафиолетовое излучение УФА-1 диапазона, длинноволновое инфракрасное излучение. Опыт применения длинноволнового инфракрасного излучения при ограниченной склеродермии недостаточно изучен. Результатов его применения на течение данного заболевания в доступной литературе мы не нашли.

В связи с этим целью настоящего исследования было изучение влияния локального длинноволнового инфракрасного излучения от аппарата CERAGEM V3 на течение ограниченной склеродермии и оценка терапевтической эффективности данного метода в комплексе с традиционно проводимой медикаментозной терапией.

\section{Материал и методы}

Под нашим наблюдением находились 55 больных ограниченной склеродермией. Из них мужчин было 13 (23,6\%), женщин - 42 (76,4\%), возраст пациентов - от 19 до 73 лет (средний возраст 32,4 года). У большинства - 33 (60\%) - обследованных давность заболевания составляла 1-2 года. Бляшечная разновидность (с 3-24 очагами поражения) была у 47 (85,5\%) пациентов, линейная (с полосовидным очагом) у 5 (9\%), склероатрофический лихен кожи у 3 (5,5\%). Для сравнительной оценки лечения пациенты были разделены на две группы, сопоставимые по полу, возрасту и тяжести заболевания (таблица).

Пациенты контрольной группы (20 человек) получали стандартную медикаментозную терапию: антибактериальные препараты (пенициллин внутримышечно по 500 тыс. ЕД 4 раза в сутки 10 дней), протеолитические ферменты (Лонгидаза), блокаторы кальциевых каналов (нифедипин), антиоксиданты (Аевит), ангиопротектор и корректор микроциркуляции для местного применения (троксерутин), а также аппликации

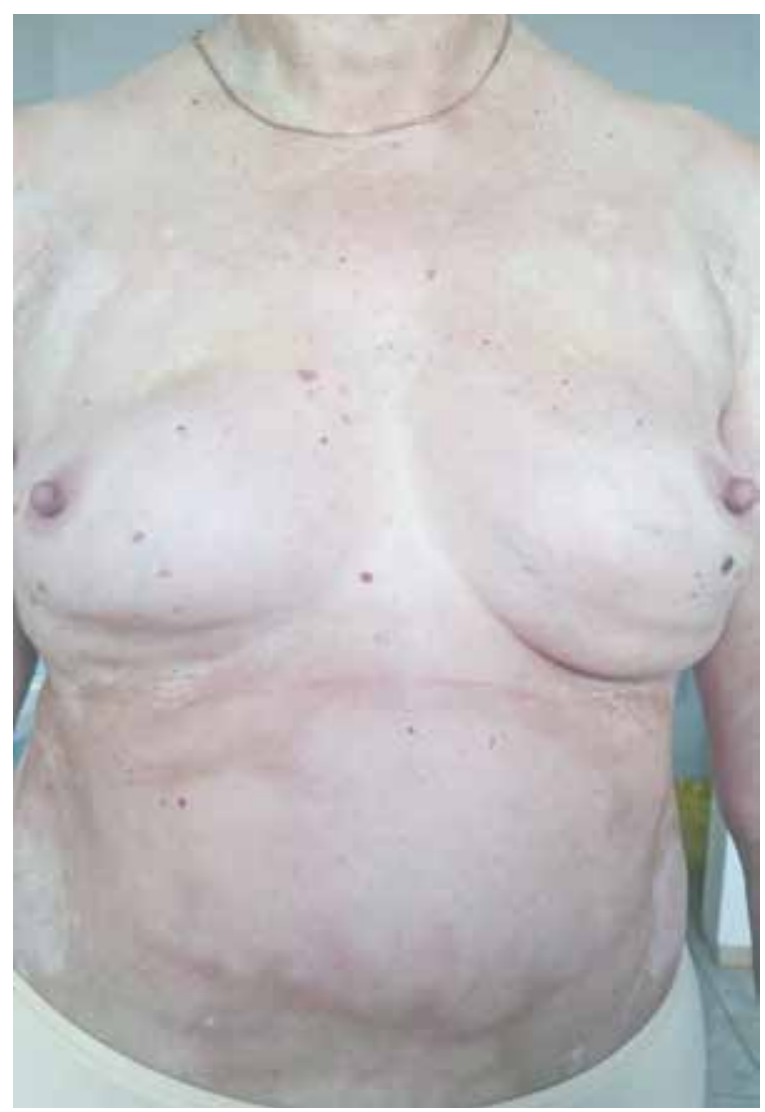

Рис. 1. Распространенная бляшечная склеродермия, генерализованный процесс
Секирин Алексей Борисович - канд. мед. наук, руководитель отделения физиотерапии и реабилитации

Смирнова Светлана Николаевна - канд. мед. наук, доцент кафедры медицинской реабилитации и физиотерапии факультета усовершенствования врачей, заведующая отделением физиотерапии и реабилитации

Сухова Татьяна

Евгеньевна - д-р мед. наук, ст. науч. сотр., отделение дерматовенерологии и дерматоонкологии'

\section{Майбродская}

Александра Евгеньевна - мл. науч. сотр., отделение физиотерапии и реабилитации $\triangle 129110$, г. Москва, ул. Щепкина, 61/2-15, Российская Федерация. Тел.: +7 (495) 6845960. E-mail:sunmyland@ rambler.ru 
Характеристика включенных в исследование больных ограниченной склеродермией

\begin{tabular}{|c|c|c|}
\hline Показатель & Основная группа & Контрольная группа \\
\hline \multicolumn{3}{|l|}{ Пол } \\
\hline мужчины & 7 & 6 \\
\hline женщины & 28 & 14 \\
\hline Возраст, годы & от 19 до 73 & от 23 до 69 \\
\hline \multicolumn{3}{|l|}{ Длительность заболевания } \\
\hline менее 1 года & 3 & 2 \\
\hline от 1 до 2 лет & 17 & 16 \\
\hline более 2 лет & 10 & 7 \\
\hline \multicolumn{3}{|l|}{ Форма заболевания } \\
\hline бляшечная & 30 & 17 \\
\hline линейная & 3 & 2 \\
\hline склероатрофический лихен & 2 & 1 \\
\hline
\end{tabular}

кортикостероидных гормонов (гидрокортизон и мометазон в форме мази).

Основная группа включала 35 пациентов, которым на фоне стандартной медикаментозной терапии проводилось локальное длинноволновое инфракрасное облучение (длина волны 4-16 мкм) очагов склеродермии. Облучение осуществляли внешними прожекторами от аппарата CERAGEM V3 (фирма CERAGEM Co., Ltd, Южная Корея). Процедуры проводились 1 раз в сутки 10 дней.

Распространенность и выраженность патологического процесса оценивали с помощью модифицированной методики балльной системы mRODNAN Skin Score. Изучали динамику интенсивности отека и гиперемии по периферии очага поражения, а также состояние микроциркуляторного русла на аппарате «Спектротест».

Подготовка и обработка статистических данных проводились с использованием текстового редактора Microsoft Office Word 2007 на основе оперативной системы Windows 7. Адекватность полученной статистической модели проверялась по F-критерию и t-критерию Стьюдента, при этом $\mathrm{p}<0,05$ - достоверность изменений между показателями, $\mathrm{n}$ - количество наблюдений.

Как было установлено, значение индекса mRODNAN при оценке распространенности и выраженности патологических изменений кожи в группах обследованных пациентов с ограниченной склеродермией варьировало от 2 до 17 (в среднем $6,1 \pm 0,6)$ баллов.

\section{Результаты и обсуждение}

При поступлении в отделение дерматовенерологии и дерматоонкологии у 44 (80\%) пациентов очаги склеродермии были бессимптомными, a у 11 (20\%) сопровождались незначительным зудом и ощущением стянутости кожи в очагах поражения. Через 14-20 суток после лечения эти жалобы отсутствовали. До проведения терапии у 35 пациентов наблюдались единичные очаги (1-3), у 10 пациентов - 4-5 очагов и еще у 10 - более 5 очагов поражения. Все очаги были в прогрессирующей стадии и характеризовались лилово-сиреневым венчиком по периферии (рис. 2А).

Стандартная медикаментозная терапия не обеспечила полного разрешения элементов ограниченной склеродермии, что подтверждалось результатами оценки индекса mRODNAN. До лечения его среднее значение в этой группе составляло $6,4 \pm 0,5$, после лечения - 5,3 $\pm 0,2$ ( $\mathrm{p} \geq 0,05)$. Таким образом, при медикаментозном лечении уплотнение и склероз кожи в очагах поражения снижались незначительно. С помощью метода лазерной спектрофотометрии в очагах поражения была отмечена тенденция к снижению сатурации кислорода крови с $0,87 \pm 0,01$ до $0,817 \pm 0,005$ у.е. и увеличению объема кровотока в микроциркуляторном русле с 0,086 $\pm 0,004$ до $0,1 \pm 0,003$ у.е. Эти данные свидетельствовали о сохранении в очагах поражения нарушения функции газообмена и трофики, хотя показатели микроциркуляции у пациентов контрольной группы имели тенденцию к нормализации (улучшение было недостоверным даже на 20-й день).

В основной группе (35 пациентов) после проведения комплексной терапии с применением локального длинноволнового инфракрасного излучения от аппарата CERAGEM V3 выраженность признаков воспаления значительно уменьшилась (рис. 2Б).

Среднее значение индекса mRODNAN снизилось существенно: до лечения этот 


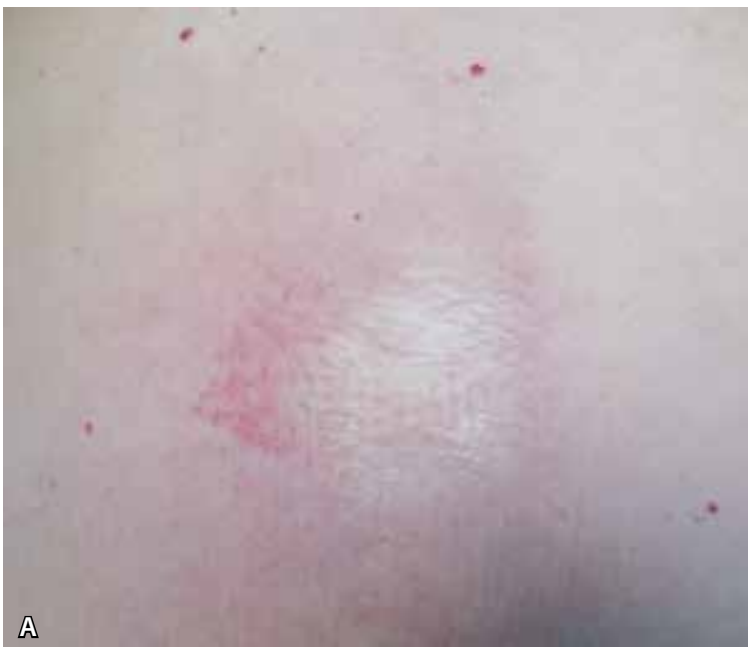

показатель составлял $6,3 \pm 0,5$, после лечения $2,2 \pm 0,1 \quad(\mathrm{p}<0,05)$, что говорило о значительном влиянии длинноволнового инфракрасного излучения на стабилизацию патологического процесса при ограниченной склеродермии. В этой группе больных побочных эффектов и нежелательных явлений отмечено не было.

При исследовании показателей лазерной спектрофотометрии в очагах патологического процесса до лечения отмечалась высокая по отношению к норме сатурация кислорода $(0,9 \pm 0,001$ у.е.) на фоне снижения показателей объема циркулирующей крови $(0,091 \pm 0,002$ у.е.), что также характерно для асептического воспаления. Через 14 дней после комплексного лечения с проведением локального длинноволнового инфракрасного излучения показатели кровотока в очаге увеличились до $0,23 \pm 0,045$ у.е. $(\mathrm{p}<0,05)$, а показатели сатурации снизились до $0,646 \pm 0,04$ у.е. $(\mathrm{p}<0,05)$, что свидетельствовало о повышении активности кислородзависимых процессов и может указывать на развитие активной репарации в очагах поражения.

Использование в комплексной терапии ограниченной склеродермии локального длинноволнового инфракрасного излучения обеспечивало более быстрое наступление клинического эффекта - на

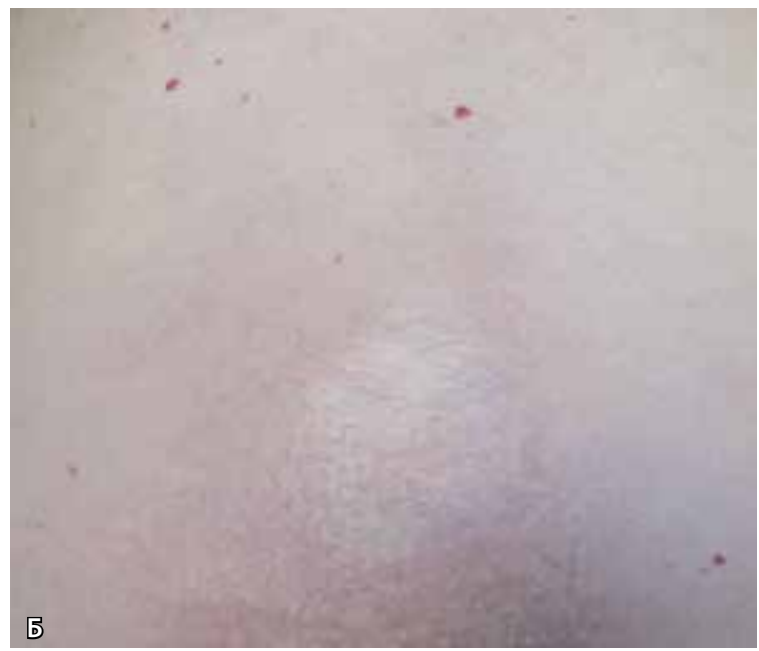

$14 \pm 2,3$ дня ( $<<0,05)$, по сравнению со стандартной медикаментозной терапией - на $20 \pm 4,2$ дня $(\mathrm{p}<0,05)$.

\section{Заключение}

Локальное длинноволновое инфракрасное излучение приводит к нормализации показателей микроциркуляции, увеличению показателя кровотока, снижению показателя сатурации кислорода в очагах поражения, а также к повышению клинической эффективности (статистически значимое снижение индекса mRODNAN: с 6,3 $\pm 0,5$ до $2,2 \pm 0,1$, $\mathrm{p}<0,05)$ и ее более быстрому наступлению. Таким образом, нами доказана клиническая эффективность применения данного физиотерапевтического метода в комплексной терапии ограниченной склеродермии.

Аппарат CERAGEM V3 подходит для использования локального длинноволнового инфракрасного излучения в комплексной терапии ограниченной склеродермии как в стационарных, так и в амбулаторных условиях.

Анализ эффективности и безопасности данного метода позволяет рекомендовать его в качестве эффективного, безопасного и современного физиотерапевтического метода лечения больных ограниченной склеродермией. ()
Рис. 2. Бляшечная склеродермия, ограниченное поражение.

А - состояние до лечения: бляшка с атрофией в центральной части и периферическим венчиком гиперемии; Б - состояние после проведения комплексной терапии с применением локального длинноволнового инфракрасного излучения: признаки активности процесса, воспаления разрешились

\section{Литература}

1.Дворников АС, Круглова ЛС, Скрипкин ЮК, Богуш Пг. Опыт применения Лонгидазы методом фотофореза у пациентов с ограниченной склеродермией. Вестник дерматологии и венерологии. 2007;(5):57-9.

2.Dharamsi JW, Victor S, Aguwa N, Ahn C, Arnett $F$, Mayes MD, Jacobe $H$. Morphea in adults and children cohort III: nested case-con- trol study - the clinical significance of autoantibodies in morphea. JAMA Dermatol. 2013;149(10):1159-65. doi: 10.1001/jamadermatol.2013.4207.

3. Chung L, Lin J, Furst DE, Fiorentino D. Systemic and localized scleroderma. Clin Dermatol. 2006;24(5):374-92. doi: 10.1016/j.clindermatol.2006.07.004
4. Toledano C, Rabhi S, Kettaneh A, Fabre B, Fardet L, Tiev KP, Cabane J. Localized scleroderma: a series of 52 patients. Eur J Intern Med. 2009;20(3):33-6. doi: 10.1016/j. ejim.2008.07.011.

5. Иванов ОЛ, ред. Кожные и венерические болезни. Справочник. М.: Медицина; 2007. $336 \mathrm{c}$. 


\section{References}

1. Dvornikov AS, Kruglova LS, Skripkin YuK, Bogush PG. Opyt primeneniya Longidazy metodom fotoforeza u patsientov s ogranichennoy sklerodermiey [Longidase administration by photophoresis to patients with circumscribed sclerodermia]. Vestnik dermatologii i venerologii. 2007;(5):57-9 (in Russian).

2. Dharamsi JW, Victor S, Aguwa N, Ahn C, Arnett $F$, Mayes MD, Jacobe $H$. Morphea in adults and children cohort III: nested case-control study - the clinical significance of autoantibodies in morphea. JAMA Dermatol. 2013;149(10):1159-65. doi: 10.1001/jamadermatol.2013.4207.

3. Chung L, Lin J, Furst DE, Fiorentino D. Systemic and localized scleroderma. Clin Dermatol. 2006;24(5):374-92. doi: 10.1016/j.clindermatol.2006.07.004.

\title{
The use of local long-wave infrared irradiation in the combination treatment of localized scleroderma
}

\author{
Sekirin A.B. • Smirnova S.N. • Sukhova T.E. ${ }^{1}$ - Maybrodskaya A.E. ${ }^{1}$
}

Background: Localized scleroderma often results in irreversible cosmetic abnormalities and if advanced, to organ dysfunction and disability. Pharmacological treatment is not effective enough and can cause adverse events and complications. Due to this fact, much attention is paid to non-medical approaches to this disorder that would be able to exert positive clinical and pathophysiological effects and potentiate the effects of medical treatment. Aim: To evaluate the effects of local long-wave infrared irradiation on the course of localized scleroderma and to assess therapeutic efficacy of this method combined with conventional pharmacological treatment. Materials and methods: Fifty five (55) patients with localized scleroderma were included into the study. The patients from the control group $(n=20)$ were administered conventional medical treatment for 14 to 20 days, including antibacterials, proteolytic enzymes, calcium antagonists, antioxidants, topical vasculoprotectors + microcirculation correctors, as well as applications of corticosteroids. The patients from the main group $(n=35)$, in addition to conventional medical treatment, were administered local long-wave infrared irradiation of the lesions (wavelength 4 to $16 \mathrm{mcm}$, once daily for 10 days). We assessed changes in edema and hyperemia on the borders of the lesion, the degree and extent of the process with a modified scoring system mRODNAN Skin Score, as well as microcirculatory vasculature by means of Spectrotest. Results: Compared to the control group, the patients with localized scleroderma who, in addition to conventional medical treatment, were administered local long-wave infrared irradiation, showed a bigger reduction of mRODNAN index $(2.2 \pm 0.1$ and $6.3 \pm 0.5$, respectively, $p<0.05)$, of oxygen saturation within the lesions $(0.646 \pm 0.04$ and $0.9 \pm 0.001$ units, $p<0.05)$ and an increase of blood flow volume in the microcirculation $(0.23 \pm 0.045$ and $0.091 \pm 0.002$ units, $p<0.05)$. They also demonstrated a quicker clinical improvement that was obvious at day $14 \pm 2.3(p<0.05)$. In the control group, the clinical improvement was seen only at day $20 \pm 4.2(p<0.05)$, with only a tendency towards a decrease in oxygen saturation within the lesions (from $0.87 \pm 0.01$ to $0.817 \pm 0.005$ ) and an increase of blood flow volume in the microcirculation (from $0.086 \pm 0.004$ to $0.1 \pm 0.003$ units). The conventional medical treatment did not result in full resolution of the local scleroderma elements, this was confirmed by the results of assessment with mRODNAN index (mean value before treatment in this group was $6.4 \pm 0.5$, after treatment, $5.3 \pm 0.2, p \geq 0.05$ ). Conclusion: The study proved the clinical efficacy of local long-wave infrared irradiation in the combination treatment of localized scleroderma. This effect was seen in the normalization of microcirculation parameters, increase of blood flow, decrease of oxygen saturation within the lesions reflecting active reparation, as well as in better clinical efficacy and more quick effect.

Key words: localized scleroderma, infrared irradiation, physical therapy, efficacy
4. Toledano C, Rabhi S, Kettaneh A, Fabre B, Fardet L, Tiev KP, Cabane J. Localized scleroderma: a series of 52 patients. Eur J Intern Med. 2009;20(3):33-6. doi: 10.1016/j. ejim.2008.07.011.

5. Ivanov $\mathrm{OL}$, editor. Kozhnye i venericheskie bolezni. Spravochnik [Skin and venereal diseases. Synopsis]. Moscow: Meditsina; 2007. 336 p. (in Russian). Sekirin Aleksey B. - MD, PhD, Head of the Physical
Therapy and Rehabilitation Department ${ }^{1}$

Smirnova Svetlana N. - MD, PhD, Associate Professor, Chair of Medical Rehabilitation and Physical Therapy, Postgraduate Training Faculty; Deputy Head of the Physical Therapy and Rehabilitation Department ${ }^{1}$

Sukhova Tat'yana E. - MD, PhD, Senior Research Fellow, Dermatovenereology and Dermato-oncology Department ${ }^{1}$

Maybrodskaya Aleksandra E. - MD, Junior Research Fellow, Physical Therapy and Rehabilitation Department ${ }^{1}$

$\triangle$ 61/2-15 Shchepkina ul., Moscow, 129110 , Russian Federation. Tel.: +7 (495) 6845960.

E-mail: sunmyland@rambler.ru 